\title{
Is the data normally distributed?
}

\author{
C. Gissane \\ School of Sport, Health and Applied Science, St Mary's University, Twickenham, Middlesex, TW1 4SX, UK \\ Tel.: +440208240 4228; Fax: +4402082404255 \\ E-mail: conor.gissane@stmarys.ac.uk.
}

\section{Introduction}

The normal distribution is the foundation of many statistical analysis techniques. These so called 'parametric methods' use the parameters of the distribution (mean, standard deviation) as part of the calculations. When data is analysed using parametric statistics, certain conditions should be met to apply those statistics correctly. One of these conditions is that data are normally distributed, and some suggest that this should be determined early in any analysis $[1,2]$. But others suggest it is unnecessary [3], as normality is not an important assumption [4] and many parametric tests are 'robust' and can deal with non-normal data distributions [3]. Yet, readers of research papers seek assurances that the data analysis is appropriate [5].

In spite of authors discussing their need, Thode [6] described approximately 400 methods to test for normality. So, many options are available to researchers which range from informal plotting through to formal hypothesis testing which tests the null hypothesis of 'the variable being examined follows a normal distribution' [1]. So, a $P$ value below a given significance level, suggests departure from normality.

Researchers need to be aware of these techniques, so that they can determine their analysis options, and to make sure the data contains no surprises. The purpose of this paper is to outline some of the techniques that can be used.

\section{Plotting data}

Healy [4] suggested that looking at your data is the best way to determine non-normality. A researcher

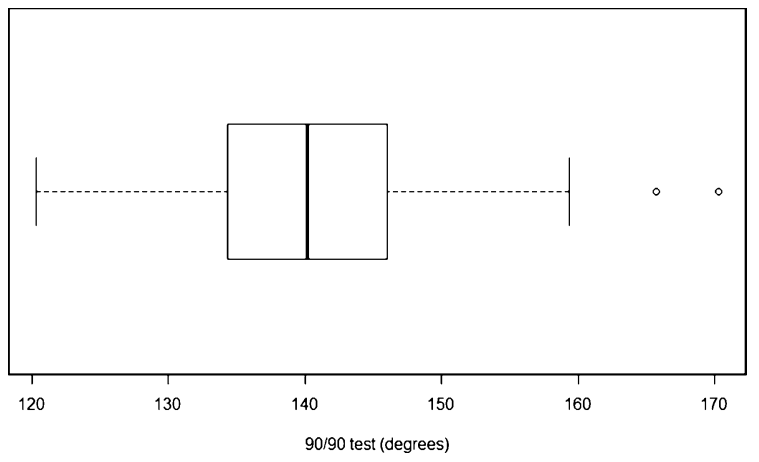

Fig. 1. Distribution of $90 / 90$ test results $(n=96)$.

should visually inspect their data first, $[1,7]$ and not doing so is according to Tukey [8], inexcusable. Henderson [7] said that the first step in analysis was to screen the data for outliers. The box plot in Fig. 1 shows a distribution, with two 'extreme' high values. The researcher can determine if they are outliers or typos.

Next, the data should be plotted in a histogram [1, 7]. This 'eye balling' enables assessing whether the data approximates to a normal distribution, and if the data has a tail. Figure 2 displays several histograms of 9090 test data scores. Selected parameters of the distributions are shown in Table 1. In Fig. 2, the distributions of the samples are different, Fig. A and B have much flatter distributions than $\mathrm{C}$ and $\mathrm{D}$, which is largely down to the respective sample sizes. Larger samples will approximate more closely to a normal distribution. Kim [9] suggests that using the histogram of the distribution may be is best in a larger sample $(n>50)$. Certainly, the two distributions with smaller 

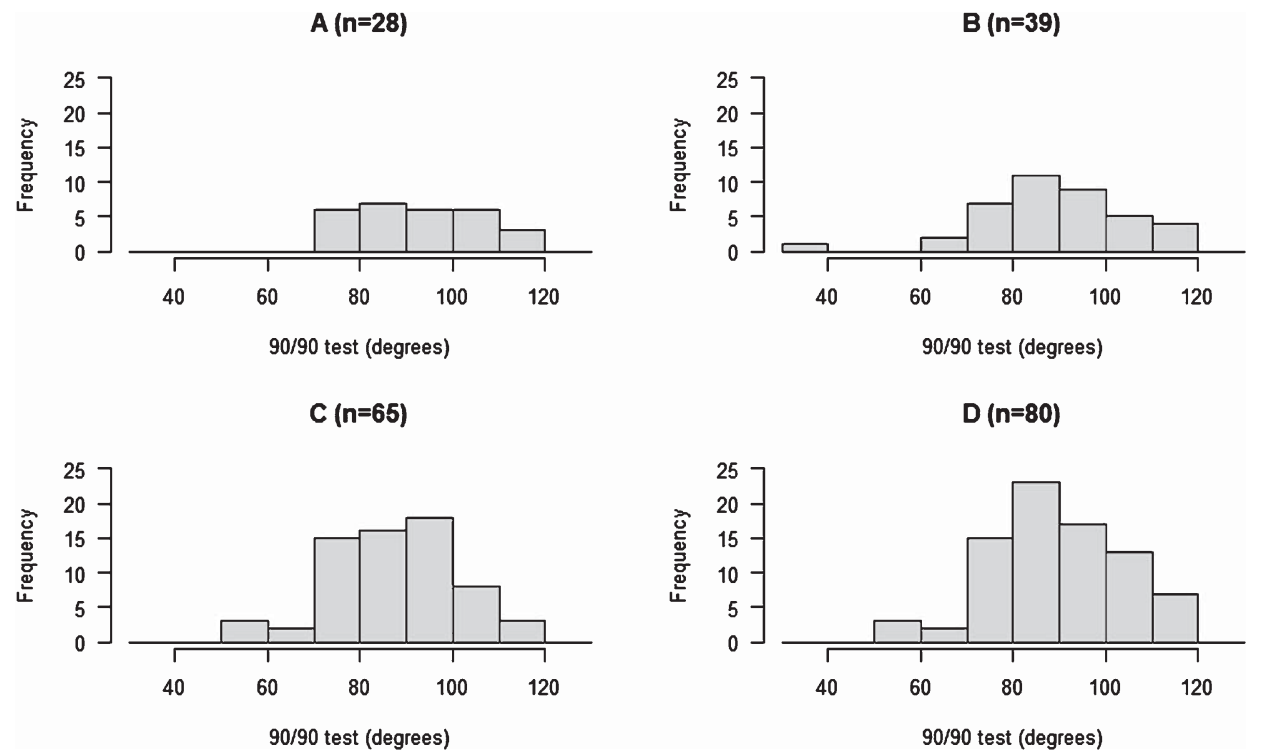

Fig. 2. Histograms of four different samples of 9090 test scores.

Table 1

Parameters of the four sample distributions

\begin{tabular}{lcccrcr}
\hline & $n$ & Mean & SD & Skew & $S_{\text {Eskew }}$ & \multicolumn{1}{c}{ Z } \\
\hline A & 28 & 92.1 & 12.4 & 0.14 & 0.463 & 0.302 \\
B & 39 & 88.3 & 16.2 & -0.71 & 0.392 & -1.810 \\
C & 65 & 86.7 & 13.9 & -0.33 & 0.304 & -1.086 \\
D & 80 & 88.9 & 13.8 & -0.39 & 0.274 & -1.424 \\
\hline
\end{tabular}

sample sizes in Fig. 1 (A \& B) are flatter and lack the distinctive shape that is beginning to emerge in Figs. $\mathrm{A}$ and $\mathrm{D}$.

Data distributions could also be examined using a graphic called a normal probability plot, sometimes know as a Q-Q probability plot. If the data approximates to a normal distribution, it should form a straight line along the upward diagonal $[1,9,10]$. Any departures from the line are evidence of a non-normal data distribution, for example Healy [4] stated that skewness can be seen if the data forms a curve. The normal probability plots for each of the samples are shown in Fig. 3. Each sample approximates quite well to the straight line. At either end of the four distributions, there is some departure from the diagonal. In each case it is not large, but it is most marked in the smaller samples (A \& B). Figure 4 shows plots for positive and negative skewed distributions. Their departure from the diagonal is marked, and they are clearly non-normal.

\section{Summary statistics}

Skewness is a measure of the asymmetry of the sample distribution [11, 12]. It is offered by many statistical packages and is also a function included in Excel [skew()]. So, it is readily accessible to researchers. For a true normal distribution the skewness parameter would be zero. A distribution is said to have a positive skew, when more data is in the right side of the distribution. A distribution is said to have a negative skew when most of the data values are on the left of the distribution. From the sample distributions in Fig. 1 and Table 1, A has a positive skew and B, C and D all have a negative skew. However, none of the values, exceed a value of \pm 2 , which is considered as the point where the distribution departs substantially from normality [9]. Another measure of departure from normality is a $\mathrm{Z}$ test as defined in Equation 1.

$$
\begin{gathered}
Z=\frac{\text { Skewness }}{S E_{\text {Skew }}} \\
S E_{\text {Skew }}=\sqrt{\frac{6}{N}}
\end{gathered}
$$

For this calculation the standard error of the skew is needed, the estimate [13] is given in Equation 2. As with any $\mathrm{Z}$ test the critical cut off at $P<0.05$ is 1.96 . 
$A(n=28)$

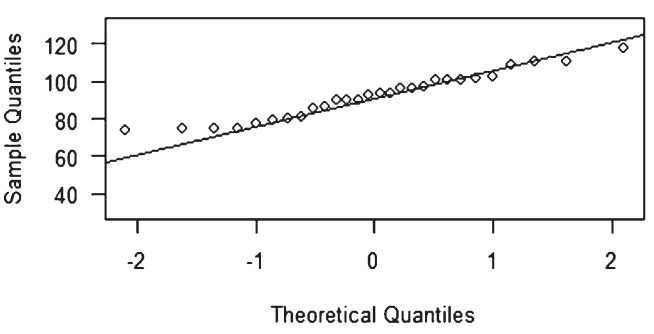

$C(n=65)$

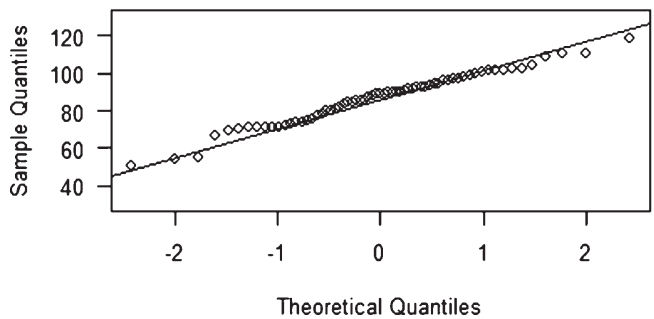

$B(n=39)$

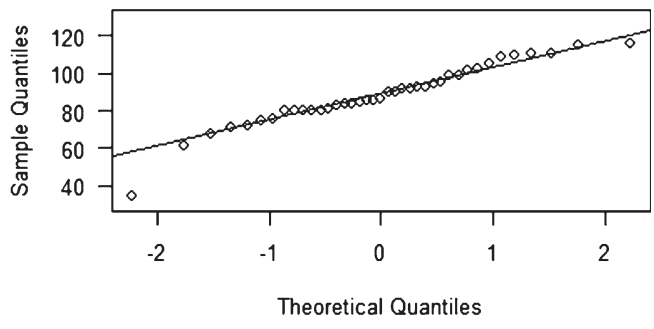

$D(n=80)$

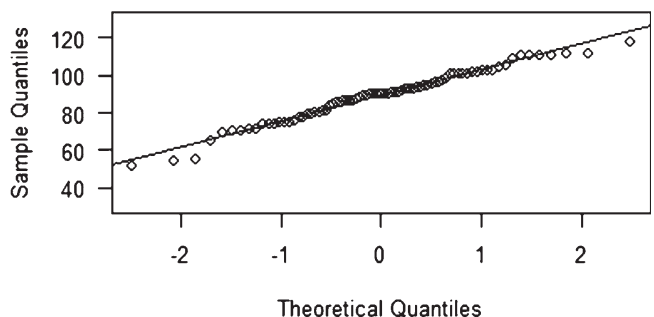

Fig. 3. Normal probability plots of four different samples of 9090 test scores.

$E(n=34)[p o s i t i v e ~ s k e w]$

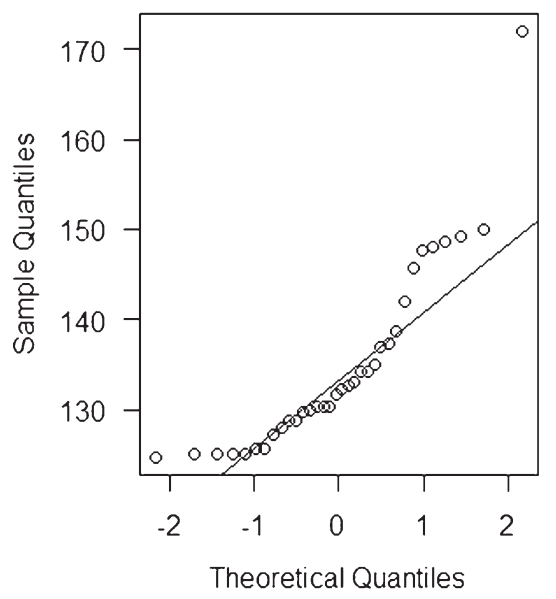

$F(n=34)$ [negative skew]

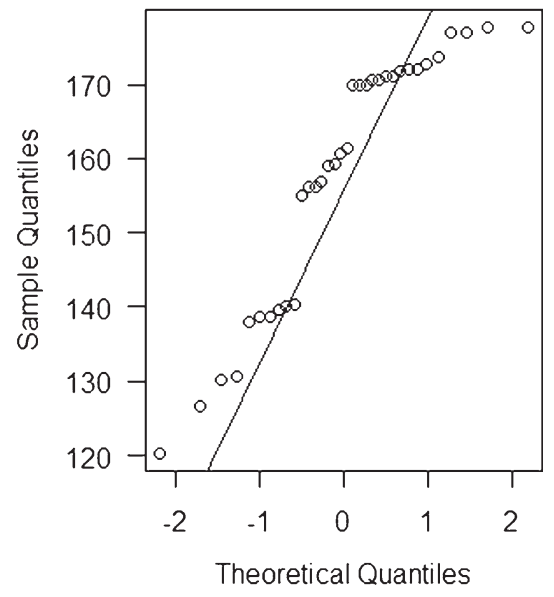

Fig. 4. Normal probability plots of a positive(E) and a negative(F) skewed distribution.

\section{Significance tests}

Researchers can apply significance tests to determine if there the data depart from normality. Two popular tests are the Shapiro-Wilk test, and the Kolmogorov-Smirnoff test. The statistics for the six samples studied are in Table 2. The results for the first four (A, B, C \& D) all recorded data above $P>0.05$ and indicate normality, whereas for samples $\mathrm{E}$ and
F, the low $P$ values indicate that the data is not normally distributed. Sainani [1] regards using these tests as optional, and if used they should accompany graphical techniques. While the Shapiro-Wilk test is more powerful than the Kolmogorov-Smirnoff test [9], it is best used with samples of under 300 [9]. With samples larger than this, it is unreliable [9] as it emphasises unimportant deviations [1]. In contrast, important deviations may be disregarded in small samples [1]. 
Table 2

Shapiro-Wilk tests for normality

\begin{tabular}{lccc}
\hline & $n$ & w & $P$ \\
\hline A & 28 & 0.959 & 0.33 \\
B & 39 & 0.946 & 0.06 \\
C & 65 & 0.981 & 0.39 \\
D & 80 & 0.978 & 0.19 \\
E & 34 & 0.832 & 0.001 \\
F & 34 & 0.877 & 0.001 \\
\hline
\end{tabular}

Just because a variable is continuous (an interval or ratio scale) does not mean the data is normally distributed. This is especially true when dealing with small samples. Any data set should be examined for normality before applying a test for differences or associations. Many techniques are available to assess normality, and researchers are urged to use a variety of methods to assess their data. Frequently in physiotherapy research, relatively small sample sizes are used $(n<50)$. Examine your data and avoid surprises.

\section{References}

[1] Sainani KL. Dealing with non-normal data. Physical Medicine and Rehabilitation 2012;4:1001-5.
[2] Lacher DA. Sampling distribution of skewness and kurtosis. Clinical Chemistry 1989;35:320.

[3] Norman G. Likert scales, levels of measurement and the 'laws' of statistics. Advances in Health Science Education 2010;15:625-32.

[4] Healy MJ. Statistics from the inside. 12. non-normal data. Archives of Diseases in Childhood 1994;70(2):158-63.

[5] Altman DG, Bland JM. Detecting skewness from summary information. BMJ 1996;313:1200.

[6] Thode HJ. Testing for normality. New York: Marcel Dekker; 2002.

[7] Henderson R. Testing experimental data for uniform normality. Clinica Chem Acta 2006;366:112-29.

[8] Tukey JW. Explaratory data analysis. Reading MA: AddisonWesley Publishing Company; 1977.

[9] Kim HY. Statistical notes for clinical researchers: Assessing normal distribution (1). Restorative Dentistry and Endodontics 2013;38:245-8.

[10] Ghasemi A, Zahediasl S. Normality tests for statistical analysis: A guide for non-statisticians. International Journal of Endocrinology and Metabolism 2012;10:486-9.

[11] Ho D, Yu CC. Descriptive statistics for modern test score distributions: Skewness, kurtosis, discreetness, and celing effects. Educational and Psychological Measurement 2015;75:365-88.

[12] Kim HY. Statistical notes for clinical researchers: Assessing normal distributions (2) using skewness and kurtosis. Restorative Dentistry and Endodontics 2013;38:52-4.

[13] Tabachnick BG, Fidell LS. Using multivariate statistics. London: Allyn and Bacon 2001. 